\title{
Perspective Article Implantation Window- still a mystery
}

\author{
Ranjani S*, Pandiyan $\mathrm{N}^{* *}$ \\ *Senior Clinical Embryologist, **Prof, Chief Consultant \& HOD, Department of Andrology \& Reproductive Medicine, \\ Chettinad Super Speciality Hospital, Chettinad Academy of Rseearch \& Education, Chennai, India.
}

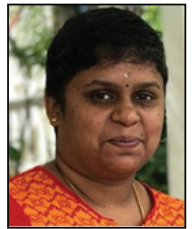

\begin{abstract}
Dr.Ranjani graduated from Government Mohan Kumaramangalam Medical College, Salem. She completed her Post Graduate Diploma in Clinical Embryology with gold medal from the Dept.of Reproductive Medicine, Chettinad Hospital and Research Institute. She is a Senior Clinical Embryologist in Department of Reproductive Medicine, Chettinad Super Specialty Hospital.
\end{abstract}

Corresponding author - Dr. Ranjani (sranjanivenkatesan@gmail.com)

Chettinad Health City Medical Journal 2020; 9(1): 2 - 4

DOI: https://doi.org/10.36503/chcmj9(1)-01

Implantation is the eventual step of assisted reproduction, whereas pregnancy is the final goal. It is a very unique phenomenon which is not decodable. The blastocyst becomes connected to the endometrium through the placenta by a secretive dialogue..$^{1-3}$ The receptivity of the endometrium during the implantation window plays an important role along with other factors.

Endometrial receptivity or implantation window assessment is the latest interest in the field of assisted reproduction. There has been a lot of ways of assessing it, the recent one being "Endometrial Receptivity Assay (ERA)".

The receptivity of the endometrium is indicated to some extent structurally and by means of changes in its secretion. Increase in the thickness, vascularization, glands becoming tortuous and secretory because of the action of hormones are noted but these are not the definitive indicators guiding us towards the embryo transfer. These changes occurring in the endometrium supposedly comes to end 7 days after the ovulation. 4

\section{How it all began?}

Noyes et al in 1950 was the first to establish morphological criteria to evaluate the endometrium. These criteria for decades remained the mainstay for defining endometrial receptivity even when it had inter- and intra-cycle variability. According to Noyes, the endometrium showed alterations in the epithelial glands from day 16 to day 20 of the menstrual cycle. The secretions of the endometrial glands were increased along with prominent subnuclear vacuolations long with decreased mitotic activity.

On the 21st day, edematous changes appears in the stroma of the Endometrium. ${ }^{5}$ Assessment of window of implantation by Noyes criteria is debatable as the accuracy of the technique has been questioned by a lot of randomized controlled trials.

\section{Pinopods}

Pinopods are the bleb like protrusions that are seen between the 19th and 21st day of menstrual cycle. These drinking feet are capable of absorbing the intrauterine fluid. This thus brings the blastocyst close to the endometrium thereby aiding the apposition, which is the first step of implantation. The presence of pinopods in the endometrium were first identified in the year $1972 .^{6-8}$

The appearance of pinopods is said to correspond to the putative window of implantation because of its limited expression for 2 days. If present, the pinopods become the preferred place of attachment for the blastocyst. The presence of pinopods is significant rather than its morphology. ${ }^{9}$ As morphology of the pinopods greatly vary, they cannot be considered as a definitive marker of window of implantation.

\section{Molecular aspects of endometrium around the implantation window}

Extensive researches have been done on the molecular aspects of the endometrium during the period of implantation window. The molecules like Cellular adhesion molecules (CAM), Integrins, Cadherins, Selectins, Immunoglobulins etc were analyzed. CAM is surface ligands that usually are glycoproteins involving in the mediation of cell-cell adhesion. Selectins are said to have a rolling effect on the blastocyst. Integrins which are expressed throughout the cycle are expressed on 20th-24th day of menstrual cycle, but only the subunit $\beta_{3}$ mRNA expression was shown to increase after day 19 which is undetected beforehand. Based on its expression pattern and epithelial localization, aV $\beta 3$ has been proposed as a potential receptor for embryonic attachment and could become an indicator of implantation window. ${ }^{10}$ 


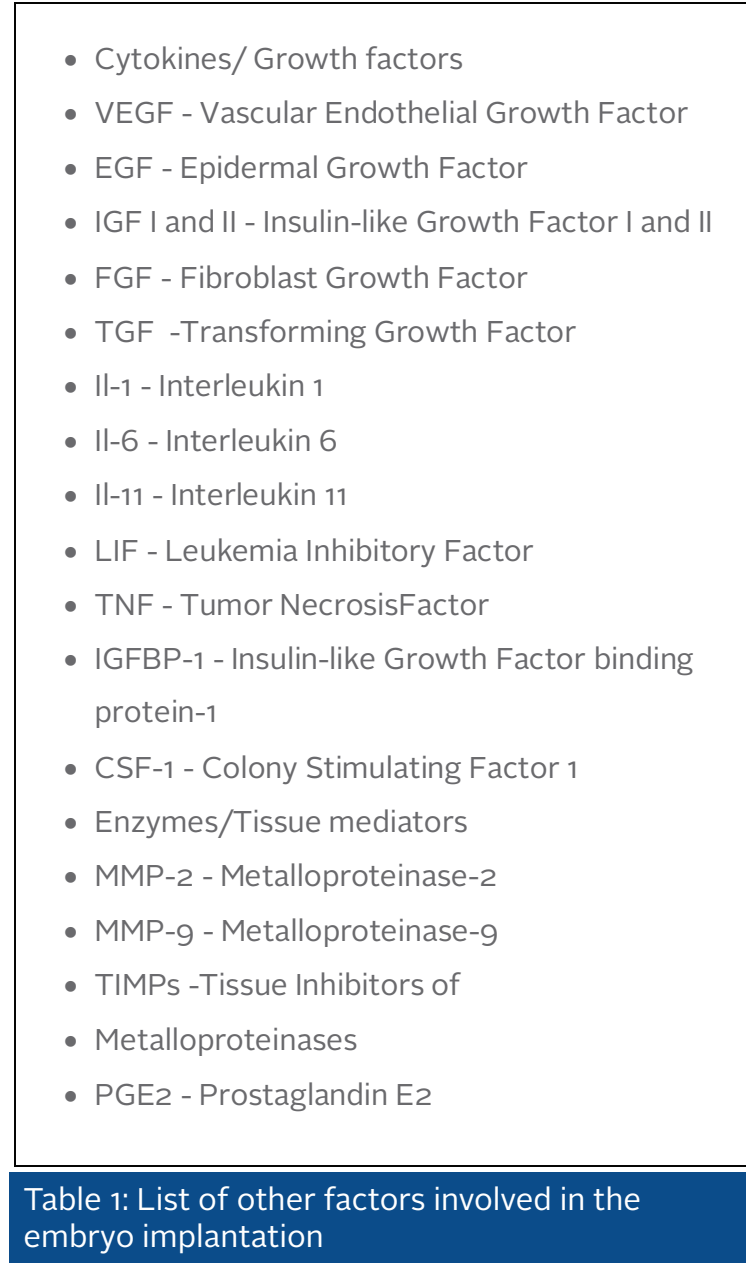

\section{Endometrial receptivity assay (ERA)}

ERA is a diagnostic test based on the genetic expression of the endometrium in which micro array transcriptomic analysis is done using the computer. This gives the status of the endometrium as receptive or non-receptive, helping us to move towards a personalized window of implantation. The endometrial tissue is biopsied after 7 days of LH surge in the previous cycle and tested for gene expression. Approximately 238 genes are studied for their expression and receptivity result is given. It is said that the test is valid for 28 to 40 weeks. The study of transcriptome of endometrial receptivity has brought to light the fact that window of implantation is not fixed as believed earlier.

Endometrial receptivity may be different even in the consecutive cycles and may vary between natural and stimulated cycle. Thus the validity of the test up to 40 weeks is questionable. It is also an invasive and expensive test which should be considered.

Therefore there is no clear idea about the implantation window and the day of embryo transfer to be done.

\section{Natural cycle vs Stimulated cycle}

The belief is that the drugs given for ovarian stimulation for recruiting follicles advances endometrial development. The genetic expression of the endo- metrium is altered due to those drugs during the implantation window period. ${ }^{11}$ Over 200 genes were expressed differentially in stimulated cycles. The pattern of expression is depended upon the type of down-regulation protocol used (agonist or antagonist) and also to the progesterone substitute for the luteal support.12 The supra-physiological levels of estrogen and progesterone during ovarian stimulation may also alter the pattern, time and duration of the receptivity. This raises a question whether the window of implantation is open or closed at the time of aiming embryo transfer.

\section{Quiet uterus - During the ovarian stimulation}

A quiet uterus facilitates the embryo attachment and implantation by aiding in invasion. ${ }^{13}$ Absence of excessive uterine contraction is found 4 days after LH surge in natural cycles which is altered in the IVF cycles. The uterine contractions are more between 4th to 6th day after HCG trigger ${ }^{14}$ in stimulated cycles which raises a question whether window of implantation is different in natural cycles and in IVF cycles.

\section{How wide is the Implantation Window?}

The two hypotheses are:

(i) The window of implantation is open for a longer period or

(ii) it is open for brief period 15

In both the hypothesis, the onset is not known. Wesley in 1991 stated that the best indicator but an indirect marker for implantation would be the serum beta HCG. In a study in which beta HCG was measured between 28th day -35th day of cycle and the time of implantation was calculated based on secretion of beta $\mathrm{hCG}>0.015 \mathrm{ng} / \mathrm{ml}$.

It was also observed that successful pregnancies occurred when the implantation was between 8th and 1oth day of ovulation and the miscarriage rates were higher when there was delay in the implantation. ${ }^{16}$

\section{Soil or seed?}

Blastocyst transfer claims to have better pregnancy rates, due to the ability to choose the better quality embryos. It is also claimed that synchrony between embryo and endometrium is better when a Day 5 embryo transfer is done, thereby improving the implantation and cumulative pregnancy rates. Also, better neonatal outcomes have been observed with blastocyst transfer.

The first pregnancy and live birth from an embryo transferred at blastocyst stage (day 5-6 after egg collection) was published in the year 1985 and 1991. Since then, a constant increase in proportion of embryo transfers at blastocyst stage has been reported which has raisedfrom $1 \%$ in 2000 to $34 \%$ in 
Upon looking into the window of implantation in detail, the questions that arose are:

- Pregnancies occur in transferring Day 7 embryos. But what is the maximum limit of day till the embryo transfer can be done?

- With recent advances blastocyst are transferred after obtaining results of PGD/PGS either in fresh cycle or frozen and transferred in the next cycle. Will endometrial receptivity array test change this routine?

- $\quad$ Can it be an IMPLANTATION DOOR??

- If implantation window is from 19th day till 24th day (ie) which corresponds to embryo day 5 to 10 , why no transfers are done on day 8 , day 9 , day 10 ?

- If stimulation affects implantation window should ET be deferred in the same cycle?

- Is Freeze all, the answer for better pregnancy rate?

- How to find out when the implantation door opens and closes?

- Will ERA give answers for everything in the future? Will there be a personalized implantation window?

- Implantation window may be small (Window) or big (door?) The day when it opens may be different for all women or is it the same? In each woman does it change with each menstrual cycle? If Stimulation alters receptivity, then how do pregnancies happen in fresh cycle embryo transfer?

- What is the implantation window in the frozen embryo transfer cycles? Since the endometrial preparation is done by the clinician, why can't the embryo transfer be done at any day in a HRT cycle?

A lot of unanswered questions exist in the implantation window. We are right in what we are doing, but the exact duration of implantation window will be done only when the embryos are transferred on Day 8,9 or 10 . Are we ethical in doing so? Window of implantation still remains a challenge for us to understand and if we can decode it then it can serve as a boon for the infertile couples.

\section{References}

1. Denker H. Implantation: A cell biological paradox. J Exp Zool. 1993;266(6):541-58.

2. Wilcox AJ, Baird DD, Weinberg CR. Time of implantation of the conceptus and loss of pregnancy. N Engl J Med. 1999;340(23):1796-9

3. Aplin JD. The cell biological basis of human implantation. Baillieres Best Pract Res Clin Obstet Gynaecol. 2000;14(5):757-64.
4. Sharma A, Kumar P. Understanding implantation window, a crucial phenomenon. J Hum Reprod Sci. 2012; 5(1): 2-6.

5. Horne AW, White JO, Lalani EN. The endometrium and embryo implantation. A receptive endometrium depends on more than hormonal influences. BMJ. 2000;321(7272):1301-2.

6. Johannisson E, Nilsson L. Scanning electron microscopic study of the human endometrium. Fertil Steril. 1972;23(9):613-25

7. Martel D, Frydman R, Glissant M. Scanning electron microscopy of postovulatory human endometrium in spontaneous cycles and cycles stimulated by hormone treatment. J Endocr. 1987;114:319-24.

8. Murphy LJ, Murphy LC, Friesen HG. Estrogen induces insulin-like growth factor-I expression in the rat uterus. Mol Endocrinol. 1987;1(7):445-50

9. Usadi RS, Murray MJ, Bagnell RC, Fritz MA, Kowalik Al, Meyer WR. Temporal and morphologic characteristics of pinopod expression across the secretory phase of the endometrial cycle in normally cycling women with proven fertility. Fertil Steril. 2003;79(4):970-4.

10. Lessey BA. Two pathways of progesterone action in the human endometrium: implications for implantation and contraception. Steroids. 2003;68(10-13):809-15.

11. Horcajadas JA, Riesewijk A, Polman J, van Os R, Pellicer A, Mosselman S et al. Effect of controlled ovarian hyperstimulation in IVF on endometrial gene expression profiles. Mol Hum Reprod. 2005 ;11(3):195-205.

12. Okada H, Tsuzuki T, Murata H. Decidualization of the human endometrium. Reprod Med Biol. 2018;17(3):220-227.

13. Bulletti C, de Ziegler Uterine contractility and embryo implantation.Curr Opin Obstet Gynecol. 2005;17(3):265-76.

14. Ayoubi JM, Epiney M, Brioschi PA, Fanchin R, Chardonnens D, de Ziegler D.Comparison of changes in uterine contraction frequency after ovulation in the menstrual cycle and in in vitro fertilization cycles. Fertil Steril. 2003;79(5):1101-5.

15. McCulloh DH, McCaffrey C, Grifo J. Biopsy on day 3 leads to delay in implantation. Fertil Steril. 2015;104(3s):e285.

16. Hearn JP, Webley GE, Gidley-Baird AA .Chorionic gonadotrophin and embryo-maternal recognition during the peri-implantation period in primates. $J$ Reprod Fertil. 1991;92(2):497-509.

17. Maheshwari A, Hamilton M, Bhattacharya S. Should we be promoting embryo transfer at blastocyst stage? Repro Bio Med . 2016; 32:142146. 